\title{
Perfil epidemiológico e distribuição demográfica do câncer de língua na região nordeste, Brasil
}

Epidemiological profile and demographic distribution of tongue cancer in the northeast region of Brazil

Perfil epidemiológico y distribución demográfica del cáncer de lengua en la región noreste de Brasil

Mauricy Nascimento FERREIRA FILHO ${ }^{1}$

Ulisses Estevam ALVES NETO ${ }^{1}$

Hellen Bandeira de Pontes SANTOS ${ }^{2}$

Andressa Cavalcanti PIRES ${ }^{2}$

${ }^{1}$ Discente do curso de Odontologia da Faculdade Nova Esperança, FACENE/FAMENE, 58067-695, João Pessoa-PB, Brasil

${ }^{2}$ Docente do curso de Odontologia da Faculdade Nova Esperança, FACENE/FAMENE, 58067-695, João Pessoa-PB, Brasil

\section{Resumo}

Introdução: A língua é uma das regiões da cavidade oral mais atingida por neoplasias malignas. Em grande parte dos casos o diagnóstico de câncer bucal é realizado tardiamente, colaborando para os altos índices de morbilidade e mortalidade dessa doença. Objetivo: Demonstrar o perfil epidemiológico e a distribuição da prevalência dos casos de câncer de língua em fumantes e ex-fumantes nos estados do nordeste brasileiro. Métodos: Trata-se de um estudo epidemiológico descritivo, transversal, realizado a partir dos dados fornecidos pelo Instituto Nacional do Câncer através dos Registros Hospitalares de Câncer, nos últimos 5 anos disponíveis, de 2013 a 2017. Todos os casos foram provenientes do Sistema Único de Saúde, separados segundo sexo e histórico de tabagismo. Os dados coletados foram analisados descritivamente. Resultados: A região do nordeste brasileiro registrou 1793 casos de câncer de língua. Os estados de Sergipe, Maranhão, Piauí apresentaram, respectivamente, os seguintes números de neoplasia na língua, 103 (5,7\%), 55 (3,1\%), e 2 (2,6\%). Já o estado da Paraíba apresentou um total de 132 (7,4\%) e Alagoas 136 (7,5\%). Os estados de Pernambuco com 651(36,3\%), Bahia com 489(27,3\%), Rio Grande do Norte e Ceará com 173(9,6\%) e 229(12,8\%) respectivamente. A análise dos dados mostrou que $72,4 \%$ dos indivíduos são homens e que o hábito de parar de fumar não impediu o aparecimento da doença. Conclusão: Existe o predomínio da referida lesão no sexo masculino, cor parda e nos indivíduos tabagistas. O estado de Pernambuco apresentou o maior número de casos de câncer de língua na região nordeste e o estado do Piauí a menor prevalência da doença.

Descritores: Neoplasias Bucais; Tabaco; Fatores de Risco.

\section{Abstract}

Introduction: The tongue is one of the regions of the oral cavity most affected by malignant neoplasms. In most cases the diagnosis of oral cancer is made late, contributing to the high rates of morbidity and mortality of this disease. Objective: Demonstrate the epidemiological profile and the distribution of the prevalence of cases of tongue cancer in smokers and exsmokers in the states of northeastern Brazil. Methods: This is a descriptive, cross-sectional epidemiological study, carried out based on data provided by Nacional Cancer Institute through Hospital Cancer Revords, in the last 5 years available. All cases came from the Unified Health System, separated according to sex and history of smoking. The collected data were descriptively analyzed. Results: Data analysis showed that northeastern region registered 1793 cases of tongue cancer. The states of Sergipe, Maranhão, Piaui presented, respectively, the following numbers of neoplasm in the tongue in the las five years: $103(5,7 \%), 55(3,1 \%)$ and $26(2,6 \%)$. The state of Paraíba presented a total of $132(7,4 \%)$ and Alagoas $136(7,5 \%)$. The states of Pernambuco with $651(36,3 \%)$ cases, Bahia $489(27,3 \%)$ Rio Grande do Norte and Ceará with $173(9,6 \%)$, $229(12,8 \%)$ simultaneously. The study also showed that $72,4 \%$ of individuals are men, and that the hábito of quitting smoking did not prevent the onset of the disease. Conclusion: There is a predominance of the referred lesion in males, brown skin and in smokers. The state of Pernambuco had the highest number of cases of tongue cancer in the northeast region and the state of Piauí had the lowest prevalence.

Descriptors: Mouth Neoplasms; Tobacco; Risk Factors.

\section{Resumen}

Introducción: la lengua es una de las regiones de la cavidad oral más afectada por las neoplasias malignas. En la mayoría de los casos, el diagnóstico de cáncer oral se hace tarde, lo que contribuye a las altas tasas de morbilidad y mortalidad. Objetivo: Demostrar el perfil epidemiológico y la distribución de la prevalencia de casos de cáncer de lengua en fumadores y ex fumadores en los estados del noreste de Brasil. Métodos: Estudio epidemiológico descriptivo, llevado a cabo utilizando los datos proporcionados por el Instituto Nacional del Cáncer a través de los Registros Hospitalarios de Cáncer, en los últimos 5 años disponibles. Todos los casos vinieron del Sistema Único de Salud separados por sexo y antecedentes de tabaquismo. Los datos recopilados se analizaron descriptivamente. Resultados: El datos mostró que la región del noreste de Brasil hubo 1793 casos de cáncer de lengua. Los estados de Sergipe, Maranhão, Piauí presentaron, respectivamente, los siguientes números de neoplasias en el lengua: $103(5,7 \%), 55(3,1 \%)$ y $26(2,6 \%)$. El estado de Paraíba 132(7,4\%) y Alagoas 136(7,5\%). Los estados de Pernambuco con 651(36,3\%) casos, Bahía 489(27,3\%), Rio Grande do Norte y Ceará, 173(9,6\%), $229(12,8 \%)$ simultáneamente. El estudio también mostró que el $72,4 \%$ de las personas son hombres, y que el hábito de dejar de fumar no previno la aparición de la enfermedad. Conclusión: Hay un predominio de la lesión referida en hombres, piel morena y en fumadores. El estado de Pernambuco tuvo el mayor número de casos de cáncer de lengua en la región noreste y el estado de Piauí tuvo la menor prevalencia.

Descriptores: Neoplasias de la Boca; Tabaco; Factores de Riesgo.

INTRODUÇÃO

Em todos os países do mundo as neoplasias malignas são causas importantes de morbidade e mortalidade, sendo que, $3 \%$ de todos os casos de câncer são encontrados na cavidade oral, tendo altas taxas de mortalidade entre todas as neoplasias malignas ${ }^{1,2}$. Entre os brasileiros o câncer bucal ocupa a quinta posição dentre as neoplasias que mais acometem homens e mulheres ${ }^{2}$.

Quanto a classificação dos cânceres que tem origem no aparelho bucal, sua divisão é dada segundo sua origem: epitelial (epitélio de revestimento e epitélio glandular), mesenquimal, hematolinfoide e odontogênico. Essas neoplasias somadas correspondem a mais de 40 tipos de câncer ${ }^{3,4}$. 
A literatura sugere que existe um perfil epidemiológico característico para câncer bucal com relação a região lingual. Evidenciando que os pacientes são do sexo masculino acima dos 40 anos idade ${ }^{5}$.

A língua é uma das regiões da cavidade oral mais atingida por neoplasias malignas ${ }^{5,6}$. A lesão se torna cada vez mais agressiva à medida que o tempo vai passando, contribuindo para um prognóstico desfavorável ${ }^{7}$. Em grande parte dos casos o diagnóstico de câncer oral é realizado tardiamente, colaborando para os altos índices de morbilidade e mortalidade dessa doença ${ }^{8}$.

Alguns fatores de risco, tais como o álcool e 0 tabaco podem desencadear 0 desenvolvimento da doença. O tabaco tem uma importante relação com 0 aparecimento de neoplasias na língua, pessoas que fazem o uso do cigarro tem um maior risco de desenvolvimento de neoplasias malignas na boca que varia entre 4 a 15 vezes mais, quando comparados a pessoas que nunca fumaram ${ }^{5,6}$.

Diante desse contexto, se faz necessário conhecer os índices de neoplasias malignas na língua, sendo assim, este estudo tem por finalidade demonstrar o perfil epidemiológico e a distribuição da prevalência dos casos de câncer de língua em fumantes e ex-fumantes nos estados do nordeste brasileiro.

MATERIAL E MÉTODO

Trata-se de um estudo epidemiológico descritivo, transversal, de base populacional com neoplasias malignas da língua ocorridos na região nordeste do Brasil, nos anos de 2013 a 2017. O presente estudo foi realizado com dados secundários por meio dos Registros Hospitalares de Câncer (RHC), disponíveis no site do Instituto Nacional de Câncer (INCA).

As variáveis estudadas foram as características do paciente: faixa etária de $(0$ a +85 anos); sexo (feminino e masculino); raça/cor (branca ; parda; negra; amarela, indígena); instrução (nenhuma; 1 a 3 anos; 4 a 7 anos; 8 a 11 anos; 12 e mais anos de estudo), bem como local de proveniências dos casos.Optou-se por selecionar os casos do SUS separando por histórico de tabagismo (exfumantes e fumantes) e escolaridade das pessoas acometidas. Os dados coletados foram analisados descritivamente. Por se tratar de dados de domínio público oferecidos no site do INCA, os sujeitos da pesquisa não são identificados, dispensando Termo de Consentimento Livre e Esclarecido (TCLE), submissão da pesquisa à Plataforma Brasil e avaliação por Comitê de Ética em Pesquisa (CEP), conforme Resolução no 46.
RESULTADOS

De acordo com a Figura 1, constatamos que, com relação ao gênero, existe predomínio do masculino (1298 casos) em comparação ao feminino (495 casos). Na análise dos cinco anos, percebemos que o percentual de incidência no sexo masculino manteve-se sem alteração significativa sendo de $72,3 \%$ dos casos. Já em relação ao gênero feminino, pode ser observado, diferenças mais acentuadas com percentagem entre $29,4 \%$ a $35,5 \%$ sendo sua média entre os anos de 27,7\%. Quanto aos estados que mais houve casos do câncer de língua, existiu um nítido predomínio da Bahia (BA) com, 27,2\% quando comparado a Pernambuco (PE) 25,2\%, Ceará (CE) com 12,8\%, Rio Grande do Norte (RN) 9,7\%. A idade mais prevalente nesses estados foi de 35 a 85 anos de idade.

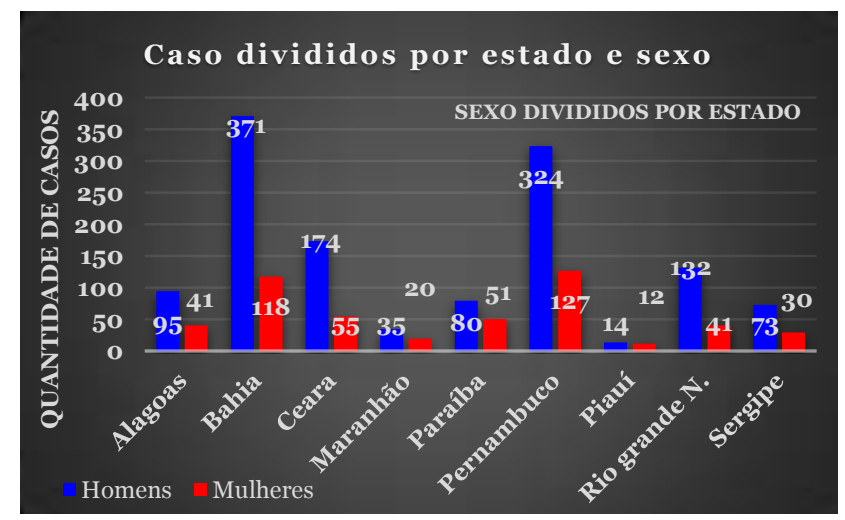

Figura 1: Número de casos por ano divididos pelo gênero nos estados do nordeste brasileiro (Fonte: dados da pesquisa).

Os estados que apresentaram menor prevalência de neoplasias foram Piauí (PI), com $1,4 \%$ dos casos; Maranhão (MA), 3\%; Sergipe (SE), com $5,7 \%$ dos casos; Paraíba (PB), 7,4\%; Alagoas (AL) $7,6 \%$ e a faixa etária mais prevalente nesses estados de 45 a 80 anos de idade. Os estados não apresentaram mudanças de grande impacto em relação à prevalência de novos casos na análise do quinquênio.

Para raça/cor (Figura 2) existiu um predomínio de pardos com $72,4 \%$ em relação aos negros $5,5 \%$, brancos com 11,7. Essas variações mantiveram-se praticamente constante no período analisado.

Quanto ao fator de risco (Figura 3), o tabagismo é muito impactante no câncer de língua sendo 1133 pacientes fumantes e exfumantes $(63,1 \%)$ de todos os anos estudados.

Já na Figura 4, que mostra o grau de escolaridade do indivíduo que foi acometido com câncer de língua mostrou que indivíduos que não chegaram a fazer no mínimo o ensino médio foram mais acometidos com essa neoplasia $69,4 \%$. 


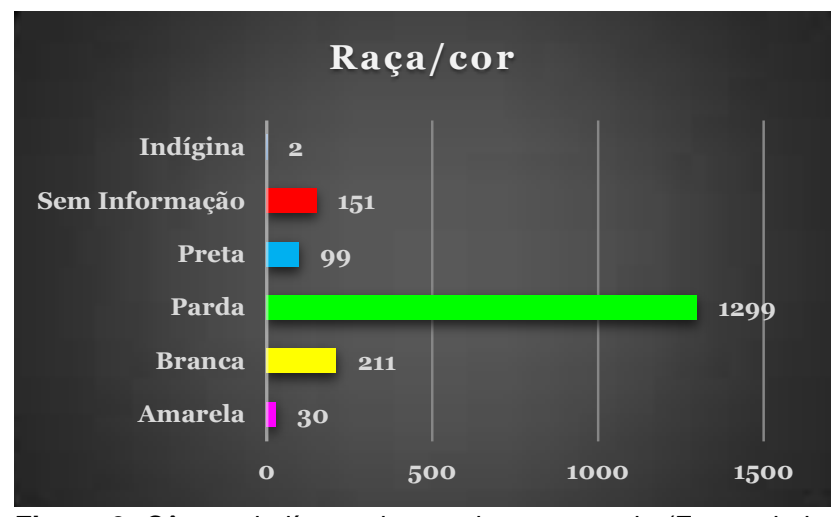

Figura 2: Câncer de língua de acordo com a etnia (Fonte: dados da pesquisa).

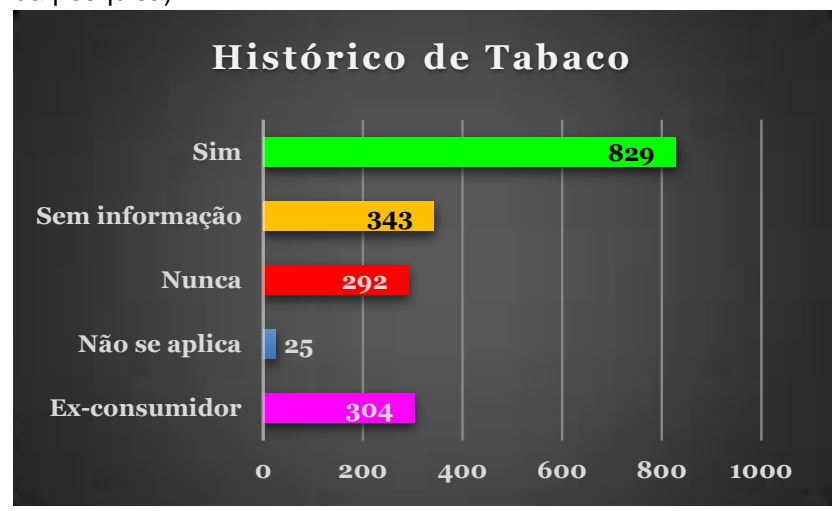

Figura 3: Histórico de tabaco de acordo com a quantidade de casos.

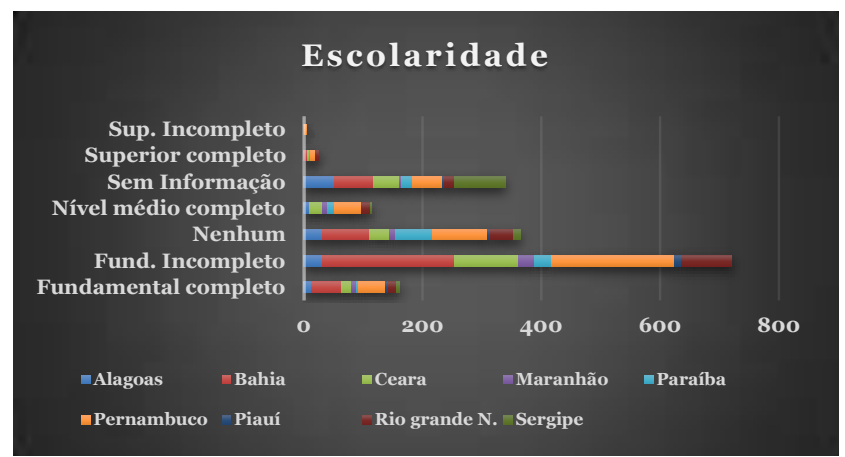

Figura 4: Distribuição do grau de escolaridade por estados (Fonte: Dados da pesquisa).

DISCUSSÃO

Em muitos países do mundo, o câncer bucal é considerado mais comum em homens do que em mulheres ${ }^{9-12}$. Isso se confirma até pelo cuidado maior que as mulheres tendem a ter com a sua saúde contribuindo contra patologias, sendo observado na proporção de homem para mulher de acordo com os resultados encontrados, são de mais de dois homens para cada mulher acometida por neoplasia maligna na região da língua, essa sendo uma das mais comuns na cavidade oral $^{7,13}$.

Estudos mostraram que o consumo de frutas e saladas atenuam o risco aumentado para o desenvolvimento de câncer bucal em fumantes, sendo este efeito protetor maior em fumantes leves ${ }^{11,12,14}$. Não está claro se isso se deve a presença das vitaminas $A, B, C$ e $E$ ou se está relacionado a outros componentes destes alimentos. O consumo de café, outras bebidas quentes, carnes grelhadas ou conservantes presentes em enlatados não parecem estar relacionado ao aumento de risco para o câncer de boca. Por outro lado, diferentes autores concordam que fatores que interferem de forma negativa no estado imunológico, como deficiências nutricionais, provavelmente favoreçam a ocorrência de câncer bucal. Esta relação não é completamente compreendida e aparentemente está envolvida na ocorrência de outras neoplasias $^{12,14,15}$

De forma geral as neoplasias bucais são mais frequentes em homens brancos da $5^{\underline{a}}$ a $6^{a}$ década de vida. Nesse sentido, os autores divergiram com os dados do presente estudo pois os indivíduos pardos ocuparam mais de setenta por cento dos casos dessa patologia, em segundo, pessoas brancas com um pouco mais de dez por cento e os negros ocupando menos de dez por cento dessa quantidade de $\operatorname{casos}^{16-19}$. O grau de escolaridade influencia diretamente nos níveis de mortalidade, uma vez que, quanto maior a escolaridade, melhor a autopercepção de saúde oral do paciente e consequentemente uma busca mais rápida por tratamento ${ }^{20,21}$.

Nesse sentido, a divergência em relação a cor da pele no presente estudo pode ser explica pela distribuição da população pelas regiões brasileiras, onde a cor parda prevalece nas regiões Norte $(68,3 \%)$, Nordeste $(64,5)$ e Centro-oeste $(49,4 \%)$ e a cor branca na região Sul $(83,6 \%)$. Isso indica que apesar da pele branca ser mais sensível aos raios solares ela não é um fator de risco considerável para o câncer de boca no Brasil, visto que a incidência de câncer de boca na região Nordeste é maior que na região $\mathrm{Sul}^{22}$.

Consumo de tabaco e de bebidas alcoólicas são os principais fatores de risco para o desenvolvimento de câncer de boca. A maior parte das pessoas acometidas pela doença se expõe ao fumo, ao álcool ou a ambos. Quando combinados, estão associados a um efeito multiplicativo $^{16-18}$. Nesse estudo o histórico de tabagismo confirma o autor citado pelo fato de a prevalência entre pessoas que fumaram na vida por serem bem mais pessoas acometidas comparadas as que nunca fumaram, de acordo com os RHC.

Apesar de novas tecnologias para doenças, a mortalidade desse tipo de patologia ainda é alta, isso se dá por conta do atraso no diagnóstico, falta do interesse do paciente em procurar profissionais ou até mesmo o profissional não ter um olhar tão aguçado em 
relação a patologias na cavidade oral, em consequência disso além de procedimentos cirúrgicos mutiladores a tecnologia utilizada para retardar células cancerígenas é muito agressivo, e o paciente vir a óbito nos primeiros cinco anos após o diagnóstico e ainda haver a possibilidade de ter metástase ${ }^{14,16}$.

Um dado interessante e que pode ser utilizado no encorajamento dos fumantes ao abandono do hábito é que o risco ao desenvolvimento de câncer reduz gradualmente frente à cessação da exposição ao tabaco, fumantes apresentam 23 vezes mais chances de desenvolver câncer do que aqueles que nunca fumaram, e os que pararam de fumar há mais de 10 anos têm um risco relativo de 1,2 , se aproximando dos indivíduos que nunca fumaram ${ }^{11,12,19}$. Diante disso, a relação de pessoas que nunca fumaram para as que já fumaram é de um pouco mais de um para um, sendo perceptível que há uma significância. A proporção de ex-fumantes para quem fuma foi de dois para um, e a de pessoas que nunca fumaram para fumantes é mais de três para um.

As distribuições de câncer de língua nos estados do Nordeste apresentam os seguintes valores em ordem crescente no estado da Bahia 489 casos, Pernambuco 451, Ceará 229, Rio Grande do Norte 173, Alagoas 136, Paraíba 131, Sergipe 102, Maranhão 55 e Piauí que apresentou o menor número, com 26 casos. 0 presente estudo teve como principal limitação o uso de dados secundários, devido ao fato que, quando acessado em ocasiões diferentes podem ter alterações nos resultados. Além de não conseguir acesso aos dados dos anos de 2018 e 2019. Esse fato pode ter acontecido devido ao preenchimento incorreto do sistema ou do preenchimento fora do prazo ordenado do Ministério da Saúde.

CONCLUSÃO

Com base nos resultados obtidos concluiu-se que o perfil epidemiológico dos pacientes diagnosticados pelas neoplasias malignas de língua caracteriza-se por pessoas do sexo masculino, cor parda e tabagistas. $O$ consumo de cigarro está atrelado ao acometimento da doença, se associada a outros fatores externos aumenta-se a chance de acometimento, embora se for cessado a exposição ao fator de risco existe possibilidade de reduzir a chance. O estado de Pernambuco apresentou o maior número de casos de câncer de língua da região nordeste e o estado do Piauí apresentou menor prevalência da doença, é sugerível que Pernambuco tenha o maior número de casos pelo fato de ter uma maior quantidade de hospitais com a finalidade de diagnosticar e tratar neoplasias malignas em relação aos do Piauí por apresentarem menos hospitais.

\section{REFERÊNCIAS}

1. Guedes JFA, Lopes JAS, Maciel VW, Nascimento PLA. Neoplasias malignas da língua: um estudo sobre a mortalidade em Pernambuco, PE [monografia]. Caruaru: Centro Universitário Tabosa de Almeida; 2017.

2. Nóbrega, TD. Avaliação clinicopatológica e sobrevida de pacientes com carcinomas epidermoides de língua, RN [monografia]. Natal: Universidade Federal do Rio Grande do Norte; 2015.

3. Cavalcanti TR. Prevalência de neoplasias malignas em região oral e maxilofacial oriundas do laboratório de patologia da Universidade Federal do Amazonas: casuística de 30 anos. 2019, AM [dissertação]. Manaus: Universidade Federal do Amazonas; 2019.

4. Konstantinos H, Roda G, Brygo A, Delaporte E, Colombel JF. Oral Cancer and Oral Precancerous Lesions in Inflammatory Bowel Diseases: A Systematic Review. J Crohn's Colitis 2015;9(11)1043-52.

5. Andrade JOM, Santos CAST, Oliveira MC. Fatores associados ao câncer de boca: um estudo de caso-controle em uma população do Nordeste do Brasil. Rev bras epidemiol. 2015;18(4):894-905.

6. Brasil. Instituto Nacional de Câncer. Falando sobre o câncer de boca [Internet]. 2002 [citado em 7 dezembro 2019]. Disponível em: https://www.inca.gov.br/tipos-de-cancer/cancerde-boca

7. Domingos PAS, Passalacqua MLC, Oliveira ALBM. Câncer bucal: um problema de saúde pública Rev Odontol Univ Cid São Paulo. 2014; 16(32):18-24.

8. Pinheiro SMS, Cardoso JP, Prado FO. Conhecimentos e diagnóstico em câncer bucal entre profissionais de Odontologia de Jequié, Bahia. Rev Bras Cancerol. 2010;56(2):195-205.

9. American Cancer Society. Oral Cavity and Oropharyngeal Cancer [Internet]. 2018 [citado em 19 de janeiro 2020]. Disponivel em: http://www.cancer.org/acs/groups/cid/document s/webcontent/003128-pdf.pdf

10. Cancer Research UK. Mouth cancer referral guidelines for dentists [internet]. 2010 [citado em 19 de janeiro 2020]. Disponivel em: http://www.cancerresearchuk.org/aboutcancer/type/mouth-cancer/about/symptoms-ofmouth--

11. Dias-Damé JL, Cesar JA, Silva SM.Time trends in smoking in an urban population: a population-based study in Southern Brazil. Cad Saúde Pública, 2011;27(11):2166-74. 
12. Enwonwu, C.O, Meeks V.I. Bionutrition and oral cancer in humans. Crit Rev Oral Biol Med. 1995;6(1):5-17.

13. Fioretti F, Bosetti C, Tavani A, Franceschi S, La Vecchia C. Risk factor for oral pharyngeal cancer in never smokers. Oral Oncol 1999;35(4):375-78.

14. Franco SR, Kowalski LP, Oliveira BV, Curado MP, Pereira RN, Silva ME, et al. Risk factors for oral cancer in Brazil: A case-control study. Int $\mathrm{J}$ Cancer 1989;43(6):992-1000.

15.Jerjes W, Upile T, Petrie A, Riskalla A, Hamdoon Z, Vourvachis $M$ et al. Clinicopathological parameters, recurrence, locoregional and distant metastasis in $115 \mathrm{~T} 1$ T2 oral squamous cell carcinoma patients. Head Neck Oncol. 2010;2:9.

16. Gandini S, Botteri E, Lodice S, Boniol H, Lowenfels AB, Maisonneuve $P$, et al. Tobacco smoking and cancer: a meta-analysis. Int $\mathrm{J}$ Cancer. 2008;122(1):155-64.

17. EUA. MAYO CLINIC. Oral cancer [Internet]. 2019 [Citado em 27 de dezembro 2019]. Dispinível em: http://www.mayoclinic.org/diseasesconditions/mouth-cancer

18. Maruccia M, Onesti MG, Parisi P, Cigna E, Troccola Um, Scuderi N. Lip cancer: a 10-year retrospective epidemiological study. Anticancer Res. 2012;32(4):1543-46.

19. Lubin J.H, Purdue H, Kelsey K, Zhang ZF, Winn $D$, Wei $Q$ et al. Total exposure and exposure rate effects for alcohol and smoking and risk of head and neck cancer: a pooled analysis of case-control studies. Am J Epidemiol. 2009;170(8):937-47.

20. Moreira MECC. Autopercepção da saúde bucal e ciência dos fatores de risco para câncer oral em idosos. Arq Ciênc Saúde. 2017;24(3):14-18.

21. Souza JLR, Araújo ACS, Nascimento FSL. O Papel do Farmacêutico na adesão de Pacientes em Uso de Antineoplásico Orais. Estácio Recife. 2019;5(2):1-12.

22. Instituto Brasileiro de Geografia e Estatística (IBGE). Distribuição da população porcor ou raça, 1999. [Internet]. [Acesso 2019 Ago30]. Disponível em: https://ww2.ibge.gov.br/home/estatistica/popula cao/condicaodevida/indicadoresminimos/tabela 1.shtm

\section{CONFLITO DE INTERESSES}

Os autores declaram não haver conflitos de interesse

\section{AUTOR PARA CORRESPONDÊNCIA}

Mauricy Nascimento Ferreira Filho

Rua Francisco de Assis Marinho, número 18

58056-250 Mangabeira - PB, Brasil

Telefone: (81) 99244-5273.

E-mail: mauricyy1258@gmail.com

Submetido em 27/06/2020

Aceito em 23/10/2020 